\title{
Declaration of Buenos Aires: position statement on the provision of critical care nursing workforce
}

Ged Williams, RN, RM, Crit. Care Cert., Grad. Cert. PSM, Bach. App. Sc. (Adv. Nursing), Grad. Cert Law, LLM (QUT), MHA (UNSW), FRCNA, FCN NSW, FACHSE, Chair, the World Federation of Critical Care Nurses

E-mail: ged.williams@maroondah.org.au

\section{BACKGROUND}

In May 2003, the World Federation of Critical Care Nurses (WFCCN) undertook a review of available national critical care nursing associations' position statements on critical care nursing workforce requirements. The current position statement aims to inform and assist critical care nursing associations, health services, governments and other interested parties in the development and provision of appropriate critical care nursing workforce requirements.

The current draft position statement aims to canvass world-wide comment and opinion on the appropriateness and acceptability of the principles and recommendations contained within it.

Final ratification of the position statement will occur at the 9th World Congress on Intensive Care and Critical Care in Buenos Aires, Argentina, in August 2005. Comments and questions on this current draft should be directed to the author at the e-mail address above.

\section{PREAMBLE}

Critical or intensive care is a complex speciality developed to serve the delicate health care needs of patients (and families) with actual or potential life threatening conditions.

The nursing workforce of critical care units requires careful planning and execution to ensure an appropriate balance and mix of staff that allows for safe and effective care while acknowledging the need to provide a learning environment for novice critical care nurses, the flexibility to respond to sudden changes in demand and the efficiency to ensure economic sustainability.

Nursing workforce planning of a critical care unit must be considered in the context of the total hospital requirement for access to critical care beds and the regional requirement for integrated and accessible critical care services across a number of hospitals in a population-defined health service.

Governments, hospital boards and professional bodies that inform and support the provision of critical care services must recognise the vital importance of providing adequately skilled, educated and available critical care nurses, doctors and other support staff, to ensure the health and safety of the most vulnerable patients in the health system.

This declaration presents guidelines universally accepted by critical care professionals, which may be adapted to meet the critical care nursing workforce and system requirements of a particular country or jurisdiction.

\section{CENTRAL PRINCIPLES}

1. All patients must be cared for in an environment that best meets their individual needs. It is the right of patients whose condition requires admission to a critical care unit to be cared for by a registered nurse. In addition, the patient must have immediate access to a registered nurse with a post-registration critical care nursing course qualification (refer to WFCCN Declaration of Madrid on the preparation of critical care nurses)

2. There should be congruence between the needs of the patient and the skills, knowledge and attributes of the nurse caring for the patient.

3. Unconscious and ventilated patients should have a minimum of one nurse to one patient. High dependency patients in a critical care unit may have a lesser nurse to patient ratio. Some patients and some environments may require more than one nurse to one patient.

4. Adequate nursing staff positions must also be in place to assist with nursing education, in-service training, quality and research programmes, management and leadership activities, and, where institutionally required, external liaison and support services beyond the confines of the critical care unit.

5. Critical care nurses should focus their labour on roles and tasks that require advanced skill and expertise in patient care. Therefore, adequate numbers of support staff should be employed to preserve the talents of critical care nurses for patient care and professional responsibilities wherever possible.

6. Flexible strategies should be employed by management to recruit, retain and remunerate expert critical care nurses at the patient bedside. Additionally, strategies should also be in place to respond to fluctuating demands on the critical care service.

\section{RECOMMENDED CRITICAL CARE NURSING WORKFORCE REQUIREMENTS}

As a minimum, the critical care unit should maintain or strive to achieve the following nursing workforce requirements:

1. Critically ill patients (clinically determined) require one registered nurse at all times. 
2. High dependency patients (clinically determined) in a critical care unit require no less than one nurse for two patients at all times.

3. Where necessary, ACCESS nurses may be employed to provide additional Assistance, Co-ordination,

Contingency (for late admission and sick staff for example), Education, Supervision and Support to a sub-set of patients and nurses in a critical care unit.

4. A critical care unit must have a dedicated head nurse (otherwise called charge nurse or similar title) to manage and lead the unit. This person must have a recognised post-registration critical care nursing course qualification.

5. $\quad$ Each shift must have a designated nurse in charge to deputise for the head nurse and to ensure direction and supervision of the unit work throughout the shift. This person must have a recognised post-registration critical care nursing course qualification.

6. A critical care unit must have a dedicated nurse educator to provide education, training and quality improvement activities for the unit nursing staff. This person must have a recognised post-registration critical care nursing course qualification.

7. Resources should be allocated to support nursing time and costs associated with quality assurance activities, nursing and team research initiatives, and education and conference attendance.

8. Adequate administrative staff, cleaning, lifting and domestic duty staff and other support personnel exist to allow nursing staff to focus on patient care and pro- fessional requirements.

9. Appropriately skilled and qualified medical doctors should be appointed and accessible to the unit for decision-making and advice at all times. A medical director should be appointed to work collaboratively with the head nurse to provide policy/protocol, direction and support.

10. Remuneration levels for nursing staff should be competitive when compared with similar professions in the country and scaled in such a way as to reward and retain qualified, experienced and senior critical care nurses.

11. Appropriate, accessible and functional levels of equipment and technology must be available and maintained to meet the demands of the expected patient load at any given time. Furthermore, nursing staff should be trained and skilled in the application of such equipment and technology.

\section{RELATED REFERENCES}

Australian College of Critical Care Nurses. (2005) Position Statement on Intensive Care Nursing Staffing [online]. Available from: www.acccn.com.au [accessed: 9 March 2005].

Pilcher T, Odell M, Bray K, Clarke S, Gardner J, Orr R \& Stirton H. (2001) Nurse-patient ratios in critical care. Nursing in Critical Care 6 (2), 59-63.

Williams GF \& Clarke T. (2001) A consensus driven method to measure the required number of intensive care nurses in Australia. Australian Critical Care 14 (3), 106-115. 